\title{
MAPPING AND ENHANCING DESIGN STUDIES WITH PSI META-THEORETIC DESIGN FRAMEWORK
}

\author{
Reich, Yoram (1); \\ Subrahmanian, Eswaran (2) \\ 1: Tel Aviv University; \\ 2: Carnegie Mellon University
}

\begin{abstract}
Design research as a field has been studied from diverse perspectives starting from product inception to their disposal. The product of these studies includes knowledge, tools, methods, processes, frameworks, approaches, and theories. The contexts of these studies are innumerable. The unit of these studies varies from individuals to organizations, using a variety of theoretical tools and methods that have fragmented the field, making it difficult to understand the map of this corpus of knowledge across this diversity.

In this paper, we propose a model-based approach that on the one hand, does not delve into the details of the design object itself, but on the other hand, unifies the description of design problem at another abstraction level. The use of this abstract framework allows for describing and comparing underlying models of published design studies using the same language to place them in the right context in which design takes place and to enable to inter-relate them, to understand the wholes and the parts of design studies.

Patterns of successful studies could be generated and used by researchers to improve the design of new studies, understand the outcome of existing studies, and plan follow-up studies.
\end{abstract}

Keywords: Research methodologies and methods, Design theory, Case study

\section{Contact:}

Reich, Yoram

Tel Aviv University

School of Mechanical Engineering

Israel

yoramr@tauex.tau.ac.il

Cite this article: Reich, Y., Subrahmanian, E. (2021) 'Mapping and Enhancing Design Studies with PSI Meta-Theoretic Design Framework', in Proceedings of the International Conference on Engineering Design (ICED21), Gothenburg, Sweden, 16-20 August 2021. DOI:10.1017/pds.2021.462 


\section{INTRODUCTION}

Design as a field has been studied from diverse perspectives starting from product or service inceptions to recycling or disposal. The outcomes of these studies include knowledge, tools, methods, processes, frameworks, approaches, and theories. The contexts of these studies are innumerable. The unit of these studies varies from individuals to groups to organizations, using a variety of theoretical tools and methods that fragment the field, making it difficult to understand the dimensions of the map of this corpus of knowledge across this diversity (Margolin, 2010; McMahon, 2012; Sanders, 2008). The range of units corresponds to the micro, meso, and macro-scale structures, each with its scope of the design problem study. The breadth of perspectives and methods is fragmented in the literature, making it difficult to get an integrated view of the field. To overcome this diversity, we need a metamodel to place them in the proper context in which design takes place and inter-relate them to understand the wholes and the parts of design studies.

The best approach to understanding the relationship between whole and parts is using a model with the same syntactic language across levels. In this paper, we propose a model-based approach that, on the one hand, does not delve into the details of the design object itself, but on the other hand, unifies the descriptions of the design problem at another abstraction level. This proposed abstract language allows for describing diverse models to understand the structure and behavior in a design context or a study. The benefit of models or theories (which are also models) for analyzing and comparing design methods has a lineage from before (e.g., Kroll et al., 2014; Reich et al., 2012; Shai et al., 2013; Sitton \& Reich, 2019; Shaked \& Reich, 2020). Nevertheless, in these studies, models were used to analyse one or two studies separately, to compare their genericity and inter-relationships, with formal models. This paper goes beyond analysing specific design methods to use our PSI model to analyse several studies that deal with actual design practice (including research practice) to explore their interrelations. Our earlier work on knowledge structure led to designing the interdisciplinary engineering knowledge genome (IEKG) for creating a formal model to bridge numerous disciplines that use a particular class of mathematical models (Reich \& Shai, 2012). In a similar spirit, we are using category theory, an abstract meta-mathematical language (Spivak, 2015), to compose the plurality of formal models used in engineering design to provide an integrated information systems infrastructure (Breiner et al., 2018, 2019). In this paper, with a similar objective of searching for patterns in design studies that span the variety of design contexts, we employ an abstract unit-level model of design and a framework to compose a multilevel model-based approach.

Multilevel modelling allows each level to employ distinct theories or ontologies. The composition of these theories across levels is critical to understand the relationship between micro, meso, and macroscale structures and behaviour. The plurality of theories and models operating at different levels of design processes is undeniable, as can be seen in the breadth of design research, just as in the design of an artifact. In this paper, the goal is to show that specific studies can be analyzed using a metalevel model to explain their outcomes and, in the process, create patterns of failures and successes.

The model we used in the study is the PSI (Problem space, Social space, Institutional space) theoretical framework (Reich \& Subrahmanian, 2019, 2020). Our experience thus far with PSI has been with analysis of diverse cases in which we participated as well as studies reported in the literature or news media. This experience has led to extending the basic PSI spaces model into a matrix (Reich \& Subrahmanian, 2020) and a network model (Reich \& Subrahmanian, 2019). PSI provided the theoretical structure to represent complex cases, understand them, as well as suggest improvements. By analyzing existing studies from the literature, we can present them with a more general frame using a unifying language to make them accessible to a broader audience. We expect, in practice, the framework to provide insights from the analysis of past studies and in planning future empirical studies.

Altogether, we demonstrate that we could "harmonize" seemingly unrelated studies through PSI, transferring insight, and consequently, the potential to improve the quality and framing of the studies. Two main advantages of this approach are that it provides a way to describe the hypothesis of the study about the problem (P), the skill base of the people performing the problem $(\mathrm{S})$, and the methods and routines they use within the structure of the organization (I). Say, for example, a traditional individual cognitive study, the problem (P), the skill set of the person $(\mathrm{S})$, and methods used by the individual (I) is the focus, and the theoretical basis is individual cognition as information processing and problem solving. Similarly, we can describe studies in distributed cognition, participant-observer 
studies, case studies, and other approaches, including activity theory. The metamodel allows us to define the study's bounds and results by identifying misalignments in PSI spaces as the cause of failure and showing how successes are well-aligned PSI models. The PSI model's main idea is that it acknowledges that different aspects of P, S, and I change over time. Hence, a reflective structure is included in the model to track their evolution and manage their alignment. We illustrate some of these properties of PSI in the analysis of design studies to point to possible templates for reporting design studies in a way that allows comparing their scope and findings. This ability of the PSI model could also lead to design experiments to study and understand the design of artifacts (P) using different configurations of skills (S) and methods and routines (I), using varied theoretical bases at different levels of the unit of observation.

The organization of the paper is as follows. Section 2 describes the methodology of this research. Section 3 briefly reviews the PSI framework. Section 4 describes the studies' analyses with PSI. Section 5 discusses the results and section 6 concludes the paper.

\section{RESEARCH METHODOLOGY}

In this study, we analyse a collection of design studies published as papers in leading refereed journals on design. We interpret the text from these studies guided by our framework and attempt to extract insight and generalizations from these interpretations. We read the chosen design study papers more than once, several months apart between readings, to make the analysis less subjective, to avoid model bias. This analysis is not a classic quantitative meta-analysis due to the studies' qualitative nature and the diversity of context of these studies. The framework used in the study allows for making predictions about the studies that could be confirmed using the outcomes they reported. Further, some studies' insights can be transferred to other studies and validated against the written report.

\subsection{Paper selection}

Paper selection is an issue when conducting literature reviews or review studies. Such selection should be representative, objective, and reproducible. Some communities worked a protocol for reporting this process called (e.g., PRISMA, http://www.prisma-statement.org/). In our case, however, there is no need to locate all or sufficiently representative sets of papers. We need to find some representatives on which we can demonstrate the approach. This entirely arbitrary collection of articles gives us sufficient evidence that our proposed approach could be valuable. We select a set of papers whose title and abstract suggest that they are concerned with actual design practice even though we contend that the approach could apply to all design research study projects. We are interested in such papers, as our analysis instrument - the PSI framework - has been validated as a framework for modelling real design situations. The selected papers are from several leading design-related journals, e.g., Research in Engineering Design, Design Studies, and Journal of Engineering Design. We are not exhaustive as the goal is to demonstrate the sufficiency of the framework to model, interpret, abstract, and even improve the narratives of the papers in these journals. Several papers were chosen from each journal, leading to a sample of about 20 papers from which we present a small selection here for lack of space.

\subsection{Meta-analysis process}

In this paper, we use the PSI framework to analyze the selected case studies. Our research methodology is different from studies that synthesize qualitative or quantitative research (see a recent analysis in Barnett-Page \& Thomas, 2009); we do not use their approach; instead, we use our metatheoretical framework. Our research interprets existing papers that report case studies and map their factors, issues, or other constructs that the papers identify onto the PSI framework. Coding was done by one or two of the authors, twice, several months apart. While not ideal practice, we can justify it by comparing the outcome of the study as explained and predicted by PSI to the actual study outcome.

Our model uses whatever information is explicitly available in the paper, including our own experience and knowledge of PSI, to provide useful insights beyond the case base of papers. The information available in all reported case studies are not at the same level of description and hence possibly compromising the completeness of each mapping. 


\section{OVERVIEW OF THE MODELING INSTRUMENT: THE PSI FRAMEWORK}

\subsection{The PSI spaces and their dimensions}

The PSI framework is a model for understanding the complexity of designing by making it rich enough to model the scope of designing yet not too complicated to be useful. It is a theoretical device to describe, analyze, explain, or change complex design situations. As designing touches upon all aspects of life, diverse disciplines such as engineering, sociology, psychology, management, and economics, have taken it as an essential subject for inquiry. These disciplines use various languages, methods, and tools leading to different perspectives. By and large, there is no integration of these perspectives and they rarely converse with each other, leading to a partial and even distorted view of designing that lends credibility to the statistics that most products conceived by designers fail by the time they reach or diffuse into the market. These partial and incomplete views of design also manifest themselves in many failures of systems of all kinds. Consequently, the first motivation of PSI is to bring together the significant aspects in designing into a single metamodel. The PSI integrates and is a framework for integrating design studies from various disciplines mentioned above and others.

The PSI framework addresses three fundamental questions about designing:

What problem is being addressed? The problem space $-\mathrm{P}$ - requires knowledge from disciplines including engineering, science, social sciences, $R \& D$, and marketing; these disciplines provide the knowledge to formulate the problem and transform it later into a product.

Who is included in designing? The social space $-\mathrm{S}$ - requires knowledge from disciplines including sociology and psychology; these disciplines allow for understanding how stakeholder personalities, capabilities, and skills, as well as the makeup of teams, determine their interactions and their ability to deal with the complexity of P and I.

How is designing performed? The institutional space - I - requiring knowledge from disciplines including economics and management; these disciplines provide the necessary background to understand different institutional cultures, structure and relationships, and the influence of problem context and market on the operation of organizations.

The PSI framework's core hypothesis is that the determinant of the success of products or services is in the alignment of the problem characteristics as defined, the knowledge in the form of social actors mobilized, and the institutional structure and routines required to operationalize the design process.

See (Reich and Subrahmanian, 2020) for significant supporting evidence for this hypothesis.

An illustration of the example of alignment uses the case of a startup company that grows into a multinational organization. Initially, the startup, composed of few friends (S), may develop a new sensor (P), in an ad hoc process (I), without any formal organizational structure or routines (I) - the PSI letters denote the space that corresponds to the information described. As the company grows, its challenge becomes much more complex - stakeholder challenges and needs change the problem to the integration of various sensors in diverse equipment $(\mathrm{P})$. The organization, now composed of hundreds of people (S), organized in a hierarchical manner (I), with offices in multiple countries, operates according to well-defined procedures and regulations (I), to deliver its contracted solutions (P). The three spaces of the company's early startup phase are aligned; the problem is relatively simple, the knowledge is focused, and an ad hoc process allows it to develop quickly. If we complicate the process, the startup model will break. In contrast, as the company grew into a multinational company, it had to address a complex problem with diverse knowledge sources in a well-organized process and institutional structure - it would not work in an ad hoc process. Alignment of all its PSI spaces is necessary for knowledge exchange and routines to match the challenge.

Each of the PS and I spaces has its own three dimensions to provide a richer characterization. The dimensions provide more expressive power to represent the complexity of design contexts. In order not to complicate the model too much, we offer three dimensions to each space. We only mention them here while detailed descriptions appear elsewhere (Reich and Subrahmanian, 2020). The characterization of the P space dimensions is (1) disciplinary complexity, (2) structural complexity, and (3) knowledge availability. The S space characterizes the social entity that addresses the design problem. This characterization uses three dimensions: (1) perspectives, (2) inclusion, and (3) capabilities/skills. The I space represents the organizational structures, rules, methods, procedures by which all the participants will be designing the product; its three-dimensional characterization are (1) ties, (2) knowledge accessibility, and (3) institutional complexity. 


\subsection{The PSI matrix model}

The PSI spaces, represented in a single layer, cannot model complex situations. For example, they cannot model the process of growing the startup company to a multinational company. To model this evolution, we need two more layers of PSI spaces, creating a matrix, shown in Table 1(a). The top layer $\mathrm{V}$ defines the vision of an entity; the middle layer A translates and implements the vision in the entity's operation, which is the lower layer O. The A layer is also responsible for reflecting on the matrix to identify misalignment of the spaces and correct them. The PSI matrix carries the properties of a single-layer PSI framework. Alignment of all the nine spaces in the matrix is necessary for the successful functioning of a design organization. To illustrate this aligning, we use a tensegrity structure, Table 1(b), as a metaphor. The bars are the spaces, and they need to be connected well so that when one changes (e.g., in the case of the startup turning to a multinational company), the other follows it. Failing to follow will tear the strings, collapsing the structure.

Table 1. (a) Three-layers PSI: from vision to an operating company in a proactive process.

The P, S, and I with subscripts are used later to denote the indexed element in the PSI matrix (b) The alignment of spaces as a tensegrity structure

(a)

\begin{tabular}{|c|c|c|c|}
\hline $\begin{array}{r}\text { Spaces/ } \\
\text { Questions }\end{array}$ & $\mid \begin{array}{l}\mathbf{P} \text { (problem } \\
\text { product) }\end{array}$ & $\begin{array}{l}\text { S } \\
\text { (social) }\end{array}$ & (institutional) \\
\hline Layers & What & Who & How \\
\hline V - Vision & $\mathrm{P}_{\mathrm{V}}$ & $\mathrm{S}_{\mathrm{V}}$ & $\mathrm{IV}_{\mathrm{V}}$ \\
\hline $\begin{array}{l}\text { A - Alignment/ - } \\
\text { Reflection }\end{array}$ & $\mathrm{P}_{\mathrm{A}}$ & $\mathrm{S}_{\mathrm{A}}$ & $\mathrm{I}_{\mathrm{A}}$ \\
\hline O - Operation & $\mathrm{P}_{\mathrm{O}}$ & $\mathrm{S}_{\mathrm{O}}$ & $\mathrm{I}_{\mathrm{O}}$ \\
\hline
\end{tabular}

(b)

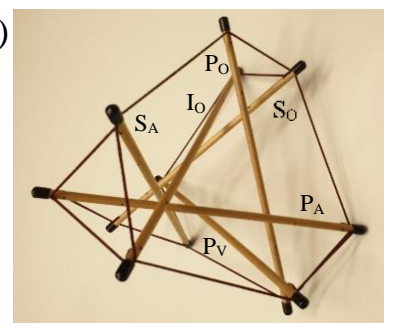

\section{INTERPRETING DESIGN RESEARCH STUDIES WITH PSI}

We now present a sample of instrumental studies to demonstrate the power of the PSI framework as a metamodeling tool for design research. In the table that follows the two examples, the numbers in the cells (spaces) refer to the numbers in bold and parenthesis in the text describing each study. The key in this representation is asking what type of answer the piece of information answers (what, who, or how) and which level it refers to. There is also a brief description of three examples.

Design study 1: Schønheyder \& Nordby (2018) (1, in Table 2) studied the evolution of design methods developed in practice as a pragmatic process to support safety-critical systems design (2). That goal or vision translated into their implementation layer through careful design: using an evolving set of mixed methods in the context of practice (3); explicitly aware of the difficulty that one of them is employed at the company and bringing other researchers (4) to serve as reviewers (5), allowing them to be reflective for changing their research methods (3). The actual study (6) involved 6 designers, 2 managers, and one co-author (7); using a particular protocol (8) that was monitored at the A layer. The study's PSI model is quite complete and well-aligned - challenges or issues complexities $(\mathrm{P})$ are matched with required skills, knowledge (S), and processes (I). The PSI hypothesis suggests this was successful research.

Table 2. PSI matrix of Schønheyder \& Nordby (2018) study: research viewpoint

\begin{tabular}{|c|c|c|c|}
\hline & $\mathbf{P}$ (problem / product) & S (social) & I (institutional) \\
\cline { 2 - 4 } & What & Who & How \\
\hline V & 2 & 1 & \\
\hline $\mathbf{A}$ & 3 & 4 & 5 \\
\hline $\mathbf{O}$ & 6 & 7 & 8 \\
\hline
\end{tabular}

If we now zoom into the study itself - modeled before as (6) in Table 2 - and model the study findings with their own PSI model, shown in Table 3; this illustrates the multilevel property of PSI. The context of safety-critical system design mandates that experience and evolution rein over innovation and revolution (1, in Table 3). Design methods (2) evolve from project to project (3) while acknowledging designers' skills (4). This evolution requires understanding (5) that the new project is different and methods may need adjustments. This understanding arises out of a 'gut feeling'; nevertheless, it and the method evolution process align the PSI spaces (5). There is also some structure in this alignment. First, it is a spiral evolution (6) that may be invoked when some aspect of the project 
context changes. The implication is that that reflection is continuous (6), suggesting that the A-layer PSI is functional all the time. The particular methods to be used in a project are determined based on its similarity with other projects (6), by its participants and designers that were involved in past similar projects (7), demonstrating care in creating the S space of the A-layer to be aligned with others. In a new project (3), methods (2) are selected to align the project and the participants' skillset (4). When methods are adapted, they are tested before application (6) - a best practice in using PSI (Reich \& Subrahmanian, 2020). Altogether, the unraveled method design exemplifies PSI matrix alignment. With that frame, it may be fruitful for the company to improve its reflection capabilities (skillset of participants in $\mathrm{S}$ of A-layer - $\mathrm{S}_{\mathrm{A}}$ ) (7) to make judgments less reliant on 'gut feeling' (6).

Table 3. PSI matrix of Schønheyder \& Nordby (2018) study: designers' viewpoint

\begin{tabular}{|c|c|c|c|}
\hline & P (problem / product) & S (social) & I (institutional) \\
\cline { 2 - 4 } & What & Who & How \\
\hline V & 1 & & \\
\hline A & 5 & 7 & 6 \\
\hline O & 3 & 4 & 2 \\
\hline
\end{tabular}

The two PSI models of the research project and method design process are also aligned. Their vision is similar, and they are both aware of the context and are being reflective. A similar situation exists in the PSI model of the n-dim project (Reich and Subrahmanian, 2020). In stating the limitations of their research, the authors suggest that it may not be repeatable. As we show later, framing the research project and the method design process with PSI makes the study and its results relevant to many other studies that also deal with practice-relevant research and PSI spaces alignment. This framing considerably increases the value of the paper, making some aspects of it repeatable.

Design Study 2: Badke-Schaub \& Frankenberger (1999) described an analysis of several projects to understand sources of successes or failures through a detailed study of various factors; these factors map well to the PSI spaces. Their results include two typical cases of failures and two typical cases of successes, shown in Table 4 and Table 5, respectively. In their language, 'failure of deficient goal analysis', which is essentially framing of the design problem in the $\mathrm{P}$ space (1), was attributed to issues (misalignment) of coordination of duties (2), availability of information (both P and I spaces, 3), and the novelty of the task (4). 'Successful solution search' (1) is attributable to the local alignment of $\mathrm{S}$ and I spaces (2). Two other cases could also be analysed as (mis)alignment of the PSI spaces. Again, such framing makes this paper relevant to a broader community from the result perspective but also the methodological perspective - it describes another way to understand the relationship between the spaces and discovers fine granularity of misalignments. Badke-Schaub \& Frankenberger (1999) and their aggregation of successful and failed cases using the factors do not engage in reflections that could be used to obviate failures in the cases based on the successes (A-level activity). However, they do identify the causes of failures. Interpreting the condition of failures using PSI, we have: choice of the participants in the $\mathrm{S}$ space (experience/skills) and their composition is critical to group organization. An I space routine determined by the group structure, where the power relationships instituted by the leader in decision making exclude the ease of information flow, leads to solving the wrong problem (solution analysis) and bad solution decision. The first case described in (BadkeSchaub \& Frankenberger, 1999) can be characterized as a contested collaboration and the second as a co-operative collaboration (Sonnewald, 1995). These are just two possibilities of the types of dynamics that can be assigned to the operation of a group. The question of coordination and external time pressure is sometimes created because of billing or client requirements that lead to the release of the design before it is complete leading to many design changes and additional costs have been observed in other studies (Subrahmanian et al., 2015).

Table 4. PSI matrix of Badke-Schaub \& Frankenberger (1999): failed projects

\begin{tabular}{|l|c|c|c|}
\hline & $\mathbf{P}$ (problem / product) & S (social) & I (institutional) \\
\hline $\mathbf{V}$ & & What & How \\
\hline $\mathbf{A}$ & & & \\
\hline $\mathbf{O}$ & $1,3,4$ & & \\
\hline
\end{tabular}


When going beyond one group to multiple groups to derive the solution to the problem, intergroup dynamics (games) are affected by intragroup dynamics (Femke, 2019). PSI makes the analysis easier to look at these effects and compare them. Badke-Schaub \& Frankenberger's (1999) paper has details that can be further mapped to specific dimensions of the PSI spaces but are beyond the scope of this paper.

Table 5. PSI matrix of Badke-Schaub \& Frankenberger (1999): successful projects

\begin{tabular}{|c|c|c|c|}
\hline \multirow{2}{*}{} & P (problem / product) & S (social) & I (institutional) \\
\cline { 2 - 4 } & What & Who & How \\
\hline $\mathbf{V}$ & & & \\
\hline $\mathbf{A}$ & & & \\
\hline $\mathbf{O}$ & 1 & 2 & 2 \\
\hline
\end{tabular}

We can transfer insight from the paper by Schønheyder \& Nordby (2018) to improve the failed cases in (Badke-Schaub \& Frankenberger, 1999); specifically, the design approach researched by them and made explicit by PSI analysis could improve the alignment between the PSI spaces of another object of study. A PSI pattern from one study provides a language for improving another study.

Design Study 3: Lundmark (2018) described a participatory design project failure with its secondary outcome of participants learning both knowledge and skills. With PSI, a complete description of the project experience with its three phases includes setting a project with only $\mathrm{O}$ layer. The $\mathrm{S}$ included participants who did not have all the skills, knowledge, and responsibility to execute it. As such, this project was destined to fail, as it did. Patterns of the successful cases in study 2 would have helped set up a better project initiation. The second phase involved participants with knowledge and skills developed in the first phase; they started to be reflective, effectively building an A-layer PSI. As the project unfolded, it became clear that there were design issues of privacy and integrity that were difficult to overcome (I space). During this phase, the participants identified themselves as project members, internalizing some ownership as a prelude for the next phase. In the third phase, the project leaders left as they had spent the budget, effectively closing the project. The participants, however, continued to operate the project, learning about the reasons for past failures and gradually developing their service and improving it further. The PSI model of this part of the design is still incomplete and not aligned. Consequently, it is unclear whether the project was sustainable.

Here also, PSI provides a broader frame that may be relevant to a wider audience in addition to the focus on participation and its secondary outcomes. Specifically, when planned correctly (Reich et al., 1996), participatory design addresses the creation of an aligned PSI matrix that increases the chances of success (i.e., achieving project goals as well as allowing participants to learn new skills, thereby empowering them). This research could have also benefited from Schønheyder \& Nordby's (2018) study to understand the necessity to align the research project's PSI.

Design Study 4: Li et al. (2019) describe the development of a new development process $\left(\mathrm{I}_{\mathrm{O}}\right)$ that, in the context of developing new products with data-driven features $\left(\mathrm{P}_{\mathrm{O}}\right)$, is aware that existing practices $\left(\mathrm{I}_{\mathrm{O}}\right)$ are inadequate $\left(\mathrm{P}_{\mathrm{A}}\right)$. The study conducted a theoretical analysis of the design problem leading to a proposal of a new process $\left(\mathrm{P}_{\mathrm{A}}\right)$. From a PSI perspective, the relationships between the spaces exercised in the research were limited. The study only tests the proposed method on a single case study, where a longer-term project in the frame of (Schønheyder \& Nordby, 2018) would have had better chances to create practical impact.

Design Study 5: Petersson \& Lundberg (2018) discussed developing an ideation method $\left(\mathrm{I}_{\mathrm{O}}\right)$ for a particular context and products $\left(\mathrm{P}_{\mathrm{O}}\right)$, for design professionals $\left(\mathrm{S}_{\mathrm{O}}\right)$ through carefully crafted action research $\left(\mathrm{I}_{\mathrm{A}}\right)$. The development, essentially an A-layer activity $\left(\mathrm{P}_{\mathrm{A}}\right)$, observed prior design practice $\left(\mathrm{I}_{\mathrm{O}}\right)$ and ideation methods $\left(\mathrm{I}_{\mathrm{O}}\right)$ and developed a new process $\left(\mathrm{I}_{\mathrm{O}}\right)$ with diverse stakeholders coming from different organizations with a potentially different culture and agenda $\left(\mathrm{S}_{\mathrm{A}}\right)$. The method was prototyped and refined $\left(I_{A}\right)$ before placing it in practice, as in (Schønheyder \& Nordby, 2018), a best practice in using PSI (Reich \& Subrahmanian, 2020. The description of the method design project is a complete, aligned PSI. It also contains details that could be used for a richer PSI model, including a multilevel network model.

\section{DISCUSSION}

If one were to read the five papers we analysed by themselves, it is almost impossible to connect them as they have different goals that are foci of those projects. Consequently, one can hardly learn from 
one study to the other. However, through theory-based modelling of the papers with PSI, patterns emerge that could transfer knowledge between the cases described in these papers. Modelling a case with a general framework allows placing it in a broader context. That context reveals relationships and additional aspects, not considered or even hidden in that context. Note that in this analysis, we refer to the papers' cases and not to the research processes; those would require a more extended analysis. Nevertheless, cases that reported successful practices (e.g., 1 and 5) could be consulted for studies where the explicit goal of the study is to impact practice and not just study practice.

For example, design study 1 studies the process of changing the methods from experience gained from previous to new projects. PSI analysis can identify the different components of the paper and the study of the process explicitly. In design study 2, the problem of determining success (denote by $2 \mathrm{~S}$ ) and failure (denote $\mathrm{S}_{\mathrm{F}}$ ) in projects is the focus. The paper uses identified factors to present a narrative and a model. Using this information as the basis for PSI coding, we were able to discern the causes of failures and successes through their respective PSI models (Table 4 and Table 5). We note that the details of design study 2 could map to even more detailed dimensions of the PSI framework than we have presented here due to space limitations; such details could enrich our capability to derive useful patterns. The insight from the successful cases $\left(2_{S}\right)$ could have been used to improve the failed cases $\left(\mathrm{S}_{\mathrm{F}}\right)$, but moreover, the patterns from study 1 could be used to avoid the failures and even improve the successes. These insights from study patterns are depicted in Figure 1. The numbers denote the studies as we numbered them, and the arrows represent that insight could transfer from one study to improve another. Furthermore, the analysis allows us to create classes of studies by their PSI model similarity. Study 1 and 5 are very similar in their PSI except that the PSI of the research study of 1 is more elaborate than the one of 5; it has an A-layer reflecting on the study and allows for introducing new research methods if necessary. Such research process may have been exercised in study 5 , but it is not reported in the paper. Placing studies 1 and 5 or 4 and $2 \mathrm{~s}$ in the same classes allows them to share insight about all their aspects, potentially improving one another even beyond what is explicitly modeled by PSI.

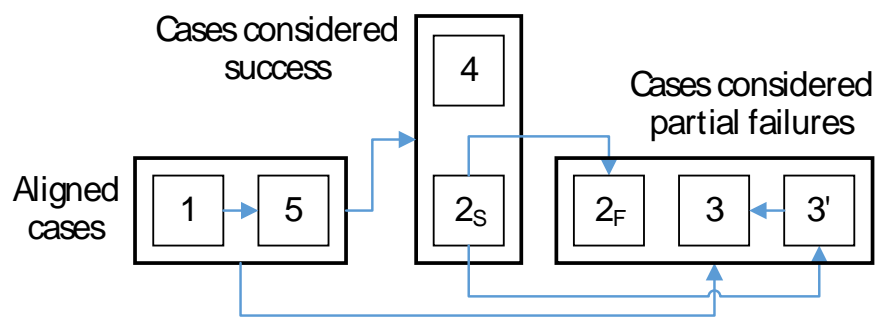

Figure 1. Insights from study patterns that could transfer from one study to another

In design study 3, PSI modelling shows when S space was restrictive and was directed by the leader without enough knowledge to define the problem $(\mathrm{P})$, resulting in failure. A subsequent change in $\mathrm{S}$ space occurred with the participants actively changing the $\mathrm{P}$ and $\mathrm{I}$ of the project. The need for the shift was learnt from the earlier failed attempt. In a sense, the departure of the leader of the project allowed the participants to reconstitute themselves based on what they learnt. If we consider the different phases in study 3 as distinct projects then the second phase, denoted by $3^{\prime}$ could have informed 3 , as shown in Figure 1. In this case, as in case 1, a failure led to a reflection that led to creating new processes and methods. The process of this case reaffirms the critical role of the A-layer in ensuring project success, and a proper set up of the case can be achieved using insight from studies 1 and 5 .

We modelled about 20 cases with PSI matrices from their reported papers. These PSI matrices provide a way to characterize the different studies in terms of what is written. As the three spaces interact and evolve at different layers to address misalignments, each design study represents a unit of design activity that is studied. In the papers, we took all of them to imply a team as the unit of analysis, even if it does not explicitly state the same in the papers. Case studies of unsuccessful and successful alignments as patterns of designing expressed as PSI matrices provide us a way to compare and contrast the studies at a high level of abstraction. The ability to analyse across research studies provides value for leveraging previous studies to explore gaps to improve them or frame new experimental or industrial studies.

The other question that could arise is, how can we deal with misalignments? What are they? What can PSI say about it? The misalignments come from not taking into account the relationship between the 
three dimensions of design in the P, S, and I spaces in all 3 layers; and alignments come from careful orchestration of the PSI at the reflective (A) level. Such reflection could lead to modifying all aspects of the PSI matrix, including a redesign of the routines and methods for the $\mathrm{I}_{\mathrm{O}}$, changing the makeup of the $\mathrm{S}_{\mathrm{O}}$ space, or even modify the problem $\left(\mathrm{P}_{\mathrm{O}}\right)$. This was the case in case studies 1 and 3. Reflection could also change the vision of the organization $\left(\mathrm{P}_{\mathrm{V}}\right)$ or the process of defining it $\left(\mathrm{I}_{\mathrm{V}}\right)$; it could also change how reflection is done $\left(\mathrm{I}_{\mathrm{A}}\right)$ and the composition of the reflecting team $\left(\mathrm{S}_{\mathrm{A}}\right)$.

In the development of design science, we have to be directly concerned with the practice that spans the wide scale of problems, skills and knowledge availability, methods, and protocols with varied institutional structures and contexts. To capture this diversity, we need a model at a high enough level of abstraction. We have shown that PSI can fulfill this function and can connect and provide value to disparate design case studies.

Due to space constraints, we have included only a few analyses of papers in detail but, we have done more of them and we continue to analyse such studies. This study has limitations that were mentioned in the research methodology section and elsewhere. In the future, we intend to pursue the following activities to account for the study limitations: obtain feedback from studies' authors on our PSI analyses, validating our models; develop a coding scheme, teach it, and evaluate its reliability with independent researchers; and increase the sample of studies including from other journals. Further, we intend to model different types of studies other than whose objective is to influence practice; and use all the richness of the PSI framework to model cases beyond the spaces, leading to detailed multilevel models.

Our study is an example of reflexive practice (Reich, 2017) - using our design methods and tools to improve our research practice. To demonstrate it further, we consider its use for our study presented in the paper. Table 6 shows our study PSI matrix. For over 30 years, our vision has been to impact design practice. From our experience, we can find clusters of general models to connect the plurality of design contexts (Reich and Subrahmanian, 2020). We mentioned such studies in the introduction. For improving design research as the present challenge, we apply the PSI as a general model that is a stepwise process where a prototype application and testing follow each step. As with other studies and PSI development included, PSI studies could revise the PSI framework and introduce additional aspects or even tools. We practice this in a parallel and complementary path to improve scientific rigor by employing other tools for increasing the reproducibility of studies (Shaked \& Reich, 2020). It is clear to us that as this project unfolds, the context may evolve and call for new skills and insight. We hope that readers will see the value in modelling their studies with PSI and will join this effort. We hope that when people perform design studies in the future, they will use PSI in their design of the study while placing it in the context of broader literature than they do today.

Table 6. This research PSI matrix

\begin{tabular}{|c|c|c|c|}
\hline & P (problem / product) & S (social) & I (institutional) \\
\hline & What & Who & How \\
\hline $\mathbf{V}$ & Impact practice & Reich \& Subrahmanian & Lifelong quest \\
\hline $\mathbf{A}$ & $\begin{array}{l}\text { Develop general models/languages to connect the } \\
\text { plurality of design contexts } \\
\text { Develop the PSI framework in a stepwise process, } \\
\text { gradually increasing model complexity to align with } \\
\text { modeling needs } \\
\text { Disseminate PSI to the community }\end{array}$ & $\begin{array}{l}\text { Reich \& Subrahmanian, } \\
\text { members of the design } \\
\text { research community }\end{array}$ & $\begin{array}{l}\text { Prototype, } \\
\text { present, teach, } \\
\text { and reflect }\end{array}$ \\
\hline $\mathbf{O}$ & $\begin{array}{l}\text { Improve design research (rigor, reproducibility, } \\
\text { relevance) by theory-driven, model-based analysis }\end{array}$ & $\begin{array}{l}\text { Reich \& Subrahmanian, } \\
\text { members of the design } \\
\text { research community }\end{array}$ & $\begin{array}{l}\text { Methodical, } \\
\text { reflective study }\end{array}$ \\
\hline
\end{tabular}

\section{CONCLUSIONS}

Our analyses of existing studies have shown how PSI provides a unified language to discuss design studies and increase their value to a broader audience, building bridges in the fragmented design discipline. Further, we found patterns we could use that describe what researchers or designers do to guide others in improving their practice. Consequently, PSI is both descriptive and prescriptive. The 
analysis presented in the paper further validates the value of PSI as a design theory and a language to describe diverse design contexts.

Two benefits have emerged from the use PSI framework. The first is, we can relate studies that deal with different design contexts with their PSI characterization providing a unified frame. The second benefit relates to cases where their PSI model is not aligned, and consequently, PSI theory predicts that these projects will fail at some point. In such cases, PSI suggests that alignment of the spaces is essential; a task of alignment will have to play a role in planning a study, during execution when the study is not progressing well, or when proposing future extensions to a study.

"Science is Organized Knowledge," is emblazoned on one of the ceilings of the Library of Congress in Washington DC. With PSI, we harmonize many disparate design studies, creating a foundation for design science.

\section{REFERENCES}

Badke-Schaub, P., \& Frankenberger, E., 1999. Analysis of design projects. Design Studies, 20(5), 465-480.

Barnett-Page, E., \& Thomas, J., 2009. Methods for the synthesis of qualitative research: a critical review. BMC medical research methodology, 9(1), 59.

Bekius, F., \& Meijer, S., 2018. The redesign process of the timetable for the Dutch railway sector: a theoretical approach. International Journal of System of Systems Engineering, 8(4), 330-345.

Bekius, F., \& Meijer, S., 2019. The redesign process of the timetable for the Dutch railway sector: a theoretical approach. Presented at the workshop on Design Theory, Design Society Special interest group in Design Theory, Jan 28-29, Paris, 2019.

Breiner, S., Jones, A. \& Subrahmanian, E., 2019. Categorical models for process planning. Computers in Industry, 112, 103124.

Breiner, S., Subrahmanian, E. \& Jones, A., 2018. Categorical foundations for system engineering. In Disciplinary convergence in systems engineering research (pp. 449-463). Springer, Cham.

Kroll, E., Le Masson, P., \& Weil, B. (2014). Steepest-first exploration with learning-based path evaluation: uncovering the design strategy of parameter analysis with $\mathrm{C}-\mathrm{K}$ theory. Research in Engineering Design, 25(4), 351-373.

Li, Y., Roy, U., \& Saltz, J. S. (2019). Towards an integrated process model for new product development with data-driven features (NPD 3). Research in Engineering Design, 1-19.

Lundmark, S., 2018. Design project failures: Outcomes and gains of participation in design. Design Studies, 59, 77-94.

Margolin, V. (2010). Doctoral education in design: problems and prospects. Design Issues, 26(3), 70-78.

McMahon, C. A. (2012). Reflections on diversity in design research. Journal of Engineering Design, 23(8), 563576.

Petersson, A. M., \& Lundberg, J. (2018). Developing an ideation method to be used in cross-functional interorganizational teams by means of action design research. Research in Engineering Design, 29(3), 433-457.

Reich, Y. (2017). The principle of reflexive practice. Design Science, 3.

Reich, Y., Hatchuel, A., Shai, O., \& Subrahmanian, E. (2012). A theoretical analysis of creativity methods in engineering design: casting and improving ASIT within C-K theory. Journal of Engineering Design, 23(2), 137-158.

Reich, Y., \& Subrahmanian, E. (2019, July). The PSI network model for studying diverse complex design scenarios. In Proceedings of the Design Society: International Conference on Engineering Design (Vol. 1, No. 1, pp. 1283-1292). Cambridge University Press.

Reich, Y., \& Subrahmanian, E. (2020). The PSI Framework and Theory of Design. IEEE Transactions on Engineering Management.

Sanders, L. (2008). An evolving map of design practice and design research. interactions, 15(6), 13-17.

Shai, O., Reich, Y., Hatchuel, A., \& Subrahmanian, E. (2013). Creativity and scientific discovery with infused design and its analysis with C-K theory. Research in Engineering Design, 24(2), 201-214.

Shaked, A., \& Reich, Y. (2020). Improving Process Descriptions in Research by Model-Based Analysis. IEEE Systems Journal.

Sitton, M., \& Reich, Y. (2019). ESE framework verification by MBSE. IEEE Systems Journal, 13(3), 2108-2117.

Spivak, D., 2017. Categories as mathematical models. Categories for the Working Philosopher, 381-401.

Schønheyder, J. F., \& Nordby, K., 2018. The use and evolution of design methods in professional design practice. Design Studies, 58, 36-62.

Sonnenwald, D. H. (1995). Contested collaboration: A descriptive model of intergroup communication in information system design. Information Processing \& Management, 31(6), 859-877.

Subrahmanian, E., Lee, C. and Granger, H. (2015). Managing and supporting product life cycle through engineering change management for a complex product. Research in Engineering Design, 26(3), 189-217. 